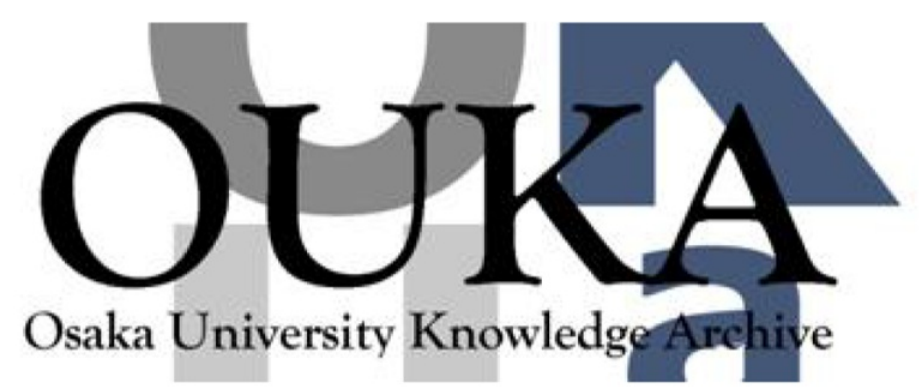

\begin{tabular}{|c|l|}
\hline Title & $\begin{array}{l}\text { Photocarrier transport in undoped } \\
\text { microcrystalline silicon studied by the } \\
\text { modulated photocurrent technique }\end{array}$ \\
\hline Author(s) & Hattori, K.; Musa, Y.; Murakami, N. et al. \\
\hline Citation & Journal of Applied Physics. 94(8) p.5071-p.5082 \\
\hline Issue Date & $2003-10-15$ \\
\hline oaire:version & VoR \\
\hline URL & https://hdl. handle. net/11094/2928 \\
\hline rights & \\
\hline Note & \\
\hline
\end{tabular}

Osaka University Knowledge Archive : OUKA

https://ir. Library. osaka-u. ac. jp/

Osaka University 


\title{
Photocarrier transport in undoped microcrystalline silicon studied by the modulated photocurrent technique
}

\author{
K. Hattori, ${ }^{\text {a) }}$ Y. Musa, N. Murakami, N. Deguchi, and H. Okamoto \\ Department of Systems Innovation, Graduate School of Engineering Science, Osaka University, Toyonaka, \\ Osaka 560-8531, Japan
}

(Received 6 June 2003; accepted 28 July 2003)

\begin{abstract}
A detailed investigation of photocarrier transport and localized states in undoped hydrogenated microcrystalline silicon $(\mu c-\mathrm{Si}: \mathrm{H})$ has been performed using the modulated photocurrent (MPC) technique. The MPC-derived drift mobility shows a systematic variation with crystalline grain size and volume fraction, as well as a distinct anisotropy. The physical implications of these observations are discussed in accordance with models that consider connectivity of crystalline grains and geometry of individual grains. The frequency spectra of MPC mobility and their dependence on excitation intensity are also analyzed in comparison with those observed for hydrogenated amorphous silicon $(a-\mathrm{Si}: \mathrm{H})$, leading to a common property of localized states that can explain the experimental results for these materials. (C) 2003 American Institute of Physics.
\end{abstract}

[DOI: $10.1063 / 1.1611638]$

\section{INTRODUCTION}

The transport properties of hydrogenated microcrystalline silicon $(\mu c-\mathrm{Si}: \mathrm{H})$ are a topic of continued interest, from the viewpoints of material physics and technological applications. The material is basically constituted of spherical or columnar Si crystallites with sizes of a few tens of nm embedded in amorphous tissue. ${ }^{1,2}$ These crystalline grains often form clusters that tend to grow normal to the substrate. At the grain boundaries and at the cluster surfaces, there may exist an increased structural disorder that brings about the occurrence of localized states of various types. Owing to such a complicated situation, the transport properties of $\mu c-\mathrm{Si}: \mathrm{H}$ are not determined or predicted straightforwardly. In particular, understanding photocarrier transport, which is crucial for solar cell applications, remains far from being complete.

Although various techniques have been utilized to investigate photocarrier transport in $\mu c-\mathrm{Si}: \mathrm{H}$ materials experimentally, ${ }^{3-10}$ some of these methods have essential limitations. Steady-state photoconductivity and photocarrier diffusion length determined from it are frequently employed to characterize transport properties. ${ }^{3,4,7,10}$ Normally, they represent the product of carrier mobility and recombination lifetime, the contributions of which are not easily decoupled in the experiments. The time-resolved experiments are capable of measuring these transport parameters separately., ${ }^{5,6,9}$ However, most of the existing tools can apply only in their specific experimental configurations, which tend to restrict the knowledge obtained from the measurements. Specifically, the possible anisotropy in photocarrier transport ${ }^{4,7}$ has not been addressed so far with a single experimental technique.

We have applied the modulated photocurrent (MPC) technique $^{11-16}$ to investigate photocarrier transport in un-

a) Author to whom correspondence should be addressed; electronic mail: hattori@ee.es.osaka-u.ac.jp doped $\mu c-\mathrm{Si}: \mathrm{H}$. The photocarrier drift mobility measured with coplanar and sandwich electrode configurations shows a systematic variation with crystalline grain size and volume fraction, as well as a distinct anisotropy. We present simple models that explain the observations reasonably well. The frequency spectra of MPC-derived mobility and their dependence on photoexcitation intensity provide a quantitative assessment of localized states that affect transport. By comparing with the results for hydrogenated amorphous silicon $(a-\mathrm{Si}: \mathrm{H})$, we deduce a common property of localized states contained in these materials.

\section{EXPERIMENT}

\section{A. Sample details}

A series of undoped $\mu c-\mathrm{Si}: \mathrm{H}$ samples was prepared by a very high frequency glow discharge at $100 \mathrm{MHz}$ with $4.5 \%$ dilution of $\mathrm{SiH}_{4}$ in hydrogen. ${ }^{17}$ The substrate temperature during the deposition was $180^{\circ} \mathrm{C}$. The film crystallinity and microstructure were varied by changing the deposition pressure between 0.08 and 1 Torr. Coplanar Al electrodes with a gap of $1 \mathrm{~mm}$ were evaporated on the top of the sample deposited on a glass substrate to investigate photocarrier transport parallel to the substrate. The sandwich structure employed for studying perpendicular transport consists of $\mathrm{ZnO} / n$-type $\quad \mu c$-Si:H/undoped, $\quad \mu c$-Si:H/n-type $\mu c-\mathrm{Si}: \mathrm{H} / \mathrm{SnO}_{2} /$ glass. The thickness of the undoped $\mu c-\mathrm{Si}: \mathrm{H}$ layer was kept constant at $2 \mu \mathrm{m}$ for all the samples. An undoped rf-glow discharge $a$-Si:H sample with coplanar electrodes was also employed in the experiment as a reference.

The structural properties of $\mu c$-Si:H samples were characterized by $\mathrm{x}$-ray diffraction and Raman scattering measurements. The x-ray diffraction lines corresponding to (111), (220), and (311) lattice planes were observed for all the samples. The average size of crystallites $\delta_{(h k l)}$ was calculated from the angular width of the $(h k l)$ diffraction peak by 


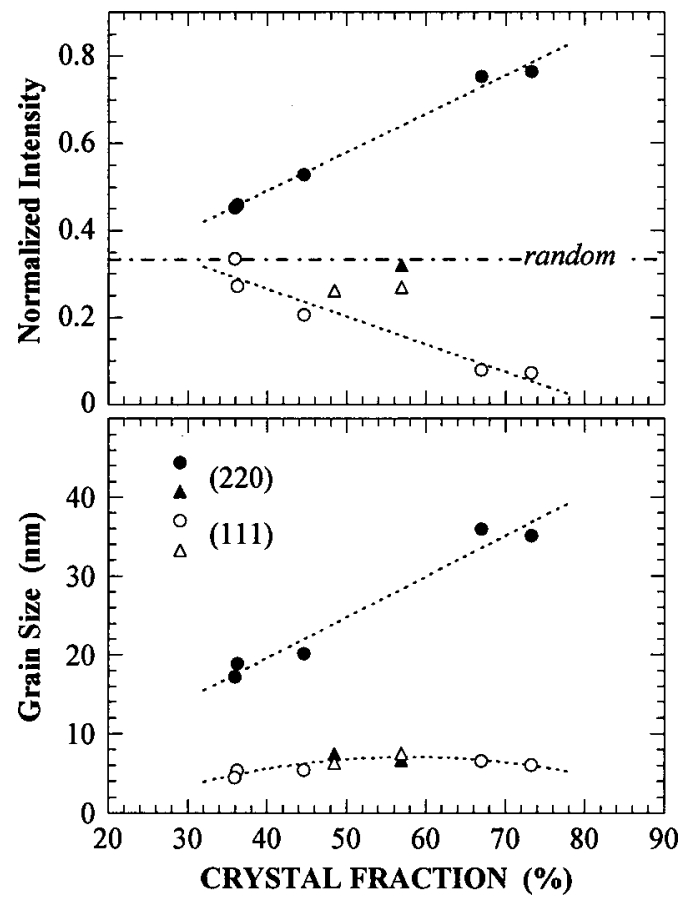

FIG. 1. Normalized x-ray diffraction intensity (top) and crystallite size (bottom) as a function of crystalline volume fraction. The chain dotted line in the top part indicates the level for random orientation. The dotted lines are drawn to guide the eye.

using Scherrer's formula. In the calculation, the instrumental broadening estimated from the measurement of pounded monocrystalline Si was taken into account. ${ }^{18}$ The crystallographic orientation was analyzed in terms of the normalized intensity defined as $p_{(h k l)}=\left[I_{(h k l)} / \hat{I}_{(h k l)}\right] / \Sigma\left[I_{(h k l)} / \hat{I}_{(h k l)}\right]$, subject to the sum rule that $\Sigma p_{(h k l)}=1$, where $I_{(h k l)}$ and $\hat{I}_{(h k l)}$ denote the diffraction intensity measured for the sample and the theoretical value for random orientation, respectively. The summation is taken over the three diffraction lines observed. Therefore, for instance, $p_{(h k l)}$ larger than $1 / 3$ means preferential growth of $(h k l)$-oriented grain. The Raman spectrum was fitted with three Gaussian bands centered around $520 \mathrm{~cm}^{-1}$ (transverse optical phonon in crystalline phase), $510 \mathrm{~cm}^{-1}$ (intermediate region), and $480 \mathrm{~cm}^{-1}$ (amorphous phase), the integrated intensities of which yielded a semiquantitative measure of the crystalline volume fraction $\chi_{c}$ in the usual manner. ${ }^{1}$

The results of structural characterization for the samples deposited directly on glass substrates are summarized graphically in Fig. 1, where the normalized intensity and the crystallite size are plotted as a function of the crystalline volume fraction. As shown in the figure, the volume fraction varies between $\chi_{c}=36 \%$ and $73 \%$. Most of the samples (indicated by circles) exhibit (220) preferential orientation, which becomes more distinct for a larger volume fraction. For this class of samples, the grain size $\delta_{(220)}$ increases from 17 to 36 $\mathrm{nm}$ monotonously with the volume fraction, while the grain size $\delta_{(111)}$ remains almost unchanged as small as $6 \mathrm{~nm}$. A few samples deposited at low pressure (indicated by triangles) have different structural properties. They contain randomly oriented and small size grains $\left(\delta_{(220)} \cong \delta_{(111)} \cong 7 \mathrm{~nm}\right)$ with a volume fraction $\chi_{c} \cong 50 \%$. The results of characterization of a set of sandwich samples are similar.

\section{B. Experimental techniques}

Here we present a brief explanation of the MPC technique. ${ }^{13-16}$ In the experiment, a semiconductor sample is illuminated homogeneously with a weakly absorbed bandgap light. The continuity equation is then represented as

$$
\frac{d}{d t} n(t)=g(t)-r(t)
$$

where $n$ is the total density of photoexcited electrons being equal to the total density of photoexcited holes due to charge neutrality, $g$ the photogeneration rate, and $r$ the recombination rate. For an excitation light varying in time sinusoidally, to first order, all the quantities appearing in Eq. (1) can be written in the form $f(t)=\bar{f}+\widetilde{f}(\omega) \exp (i \omega t)$. The modulated part of the photocarrier density is simply expressed as

$$
\widetilde{n}(\omega)=\frac{\widetilde{g}(\omega) \tau(\omega)}{1+i \omega \tau(\omega)}
$$

with the average lifetime for all the charge carriers defined as $\tau(\omega)=\widetilde{n}(\omega) / \widetilde{r}(\omega)$. The conductivity is also separated into two components such that $\sigma(t)=\bar{\sigma}+\widetilde{\sigma}(\omega) \exp (i \omega t)$. The modulated photoconductivity is formally written as

$$
\widetilde{\sigma}(\omega)=q \mu(\omega) \widetilde{n}(\omega),
$$

where $q$ denotes the unit electronic charge, and $\mu(\omega)$ is the drift mobility that represents the average mobility for all the charge carriers. On the basis of the phenomenological consideration described above, we finally define the MPC mobility as

$$
\mu_{\mathrm{MPC}}(\omega) \equiv \frac{i \omega \widetilde{\sigma}(\omega)}{q \widetilde{g}(\omega)}=\mu(\omega) \frac{i \omega \tau(\omega)}{1+i \omega \tau(\omega)},
$$

which coincides with the drift mobility $\mu(\omega)$ in the high frequency region where the condition that $\omega|\tau(\omega)| \gg 1$ is fulfilled. On the other hand, in the low-frequency region where $\omega|\tau(\omega)| \ll 1$, the MPC mobility is reduced to $i \omega \mu(\omega) \tau(\omega)$. In this extreme limit, the modulated photoconductivity $\widetilde{\sigma}(\omega)$ is in phase with the excitation $\widetilde{g}(\omega)$. In other words, the carrier system remains in a quasisteady state during modulation and thereby the transport parameters $\mu(\omega)$ and $\tau(\omega)$ become real numbers and frequency independent.

Experimentally, a sinusoidal excitation for the MPC measurements was provided by a laser diode with a wavelength of 678 or $840 \mathrm{~nm}$. The modulation frequency was varied between $\omega / 2 \pi=10 \mathrm{~Hz}$ and $40 \mathrm{MHz}$. The amplitude of modulated photoconductivity and its phase shift with respect to the excitation were detected by a standard lock-in amplifier for low frequencies $(<100 \mathrm{kHz})$ or a rf lock-in amplifier for high frequencies $(>10 \mathrm{kHz})$. The voltage applied between the electrodes was chosen in the range where the current-voltage characteristic was linear. In this article, the MPC mobility data are presented in the form $\operatorname{Re}\left[\mu_{\mathrm{MPC}}(\omega)\right]$, which enables us to clearly distinguish the high and low frequency regimes. Using the same experimental apparatus, 


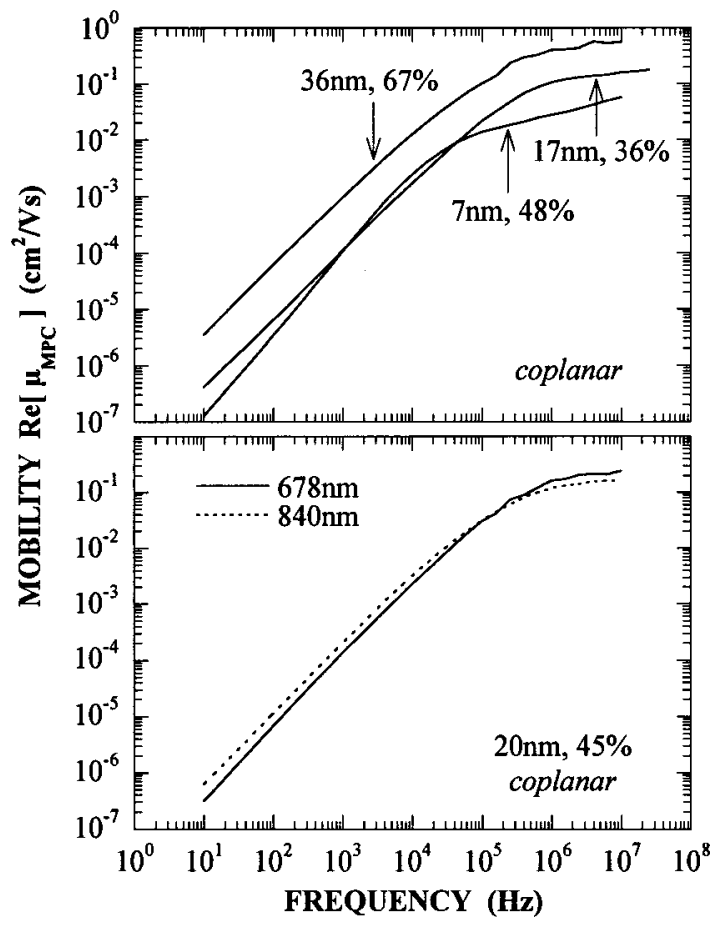

FIG. 2. Frequency spectra of MPC mobility measured in the coplanar electrode configuration for the steady-state generation rate $\bar{g}=1-2$ $\times 10^{20} \mathrm{~cm}^{-3} \mathrm{~s}^{-1}$. The top part summarizes the results obtained with $678 \mathrm{~nm}$ excitation for samples with various structural properties. The bottom part compares the results for different excitation wavelengths, 678 and $840 \mathrm{~nm}$. The crystalline grain size $\delta_{(220)}$ and volume fraction $\chi_{c}$ are indicated.

the frequency dependence of admittance under steady-state illumination was measured concomitantly. All the experiments were performed at room temperature.

\section{DRIFT MOBILITY MEASUREMENTS}

\section{A. Parallel transport}

Figure 2 displays the frequency spectra of MPC mobility measured in the coplanar electrode configuration for the steady-state photogeneration rate $\bar{g}=1-2 \times 10^{20} \mathrm{~cm}^{-3} \mathrm{~s}^{-1}$. The upper panel of the figure shows the results obtained with a $678 \mathrm{~nm}$ excitation for samples with various structural properties. The lower figure compares the results obtained for different excitation wavelengths 678 and $840 \mathrm{~nm}$. As demonstrated in the figure, the MPC mobility is almost the same for the two different excitation wavelengths. The spectra tend to plateau in the higher frequency region while in the lower frequency region they go downward with decreasing frequency. The low frequency falloff signifies a quasisteadystate transport, which is largely affected by recombination. The translation of MPC mobility into drift mobility can be therefore validated only for the high frequency branch. Figure 3 summarizes the MPC mobility measured at $10 \mathrm{MHz}$ as a function of the crystalline volume fraction. It has been confirmed in the experiment that $\operatorname{Re}\left[\mu_{\mathrm{MPC}}\right] \cong\left|\mu_{\mathrm{MPC}}\right|$ at such a high frequency. The MPC mobility for the samples containing large grains $\left(\delta_{(220)}=17-36 \mathrm{~nm}\right)$ increases with the volume fraction, while the sample comprised of small grains $\left(\delta_{(220)} \cong 7 \mathrm{~nm}\right)$ shows a lower mobility even with a relatively large volume fraction.

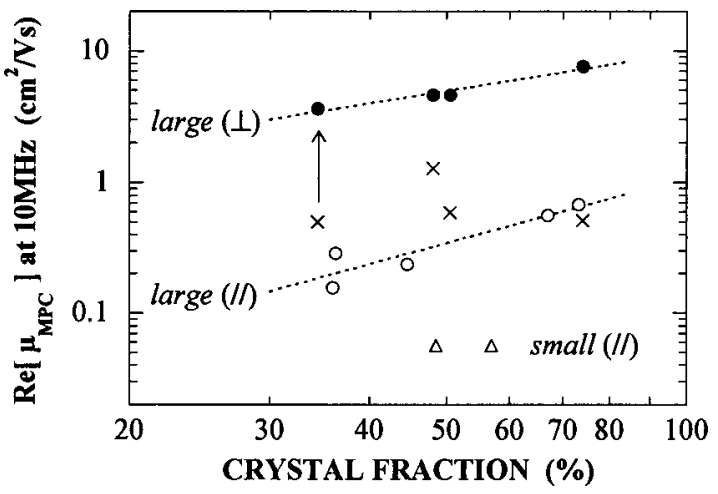

FIG. 3. The crystalline volume fraction dependence of $10 \mathrm{MHz}$ drift mobility measured in coplanar $(\|)$ and sandwich $(\perp)$ configurations. The data for samples with large crystalline grains $\left(\delta_{(220)} \geqslant 17 \mathrm{~nm}\right)$ are plotted as circles and those with small grains $\left(\delta_{(220)} \cong 7 \mathrm{~nm}\right)$ as triangles. The results of measurements for the sandwich structure $(X)$ are corrected in accordance with the equivalent circuit model $(\bullet)$. The dotted lines are drawn to guide the eye.

The $678 \mathrm{~nm}$ light, which has an energy $(1.83 \mathrm{eV})$ larger than the band gap of the crystalline $(1.11 \mathrm{eV})$ and amorphous $(1.75 \mathrm{eV})$ phases, excites carriers in both of them, whereas photoexcitation from the $840 \mathrm{~nm}(1.48 \mathrm{eV})$ light predominantly takes place in the crystalline phase. The experiments showing no discernible difference in the MPC mobility imply that photocarriers generated in the amorphous phase are mainly injected into the crystalline phase via diffusion, and hardly return to the amorphous phase due to finite band edge discontinuities between these phases. Assuming the electron drift mobility in the amorphous phase to be $1 \mathrm{~cm}^{2} / \mathrm{V} \mathrm{s},{ }^{13-15,19}$ the root-mean-square distance traveled by a diffusing electron is estimated to $160 \mathrm{~nm}$ in a time scale of $10 \mathrm{~ns}$, which corresponds to the maximum modulation frequency of the experiment. For a sufficiently high excitation, the diffusion of charge carriers becomes ambipolar and controlled by holes with a lower mobility. ${ }^{20-22}$ According to the multiple trapping model, ${ }^{19}$ the hole drift mobility is calculated to be about $5 \times 10^{-3} \mathrm{~cm}^{2} / \mathrm{Vs}$ at $10 \mathrm{~ns}$. This amounts to an ambipolar diffusion length of $10 \mathrm{~nm}$. These estimated values of diffusion length are larger than or comparable to a mean spacing between grains for our samples, which is evaluated to be $\Delta \approx 6-7 \mathrm{~nm}$ at most, based on the following relation: $\chi_{c} \approx 1 /(1+\Delta / \delta)^{3}$. The comparison of the characteristic lengths reasonably explains that photocarriers are substantially accumulated in crystalline phase.

Carrier transport in any inhomogeneous media is essentially statistical, and could be illustrated in the context of the percolation theory. ${ }^{23,24}$ A simple model for carrier transport in $\mu c-\mathrm{Si}: \mathrm{H}$ is to consider that connected crystalline grains form a percolation channel through which current flows effectively. The percolation probability that a given grain is sufficiently well connected to the rest to be available for carrier transport on a macroscopic scale increases with the volume fraction above a certain threshold. The percolationlimited transport in $\mu c$-Si:H may also depend on the dimension of grains. For example, it is likely that large size grains make grain-to-grain carrier transfer more efficient because of their larger contact area. The other important factors to be 


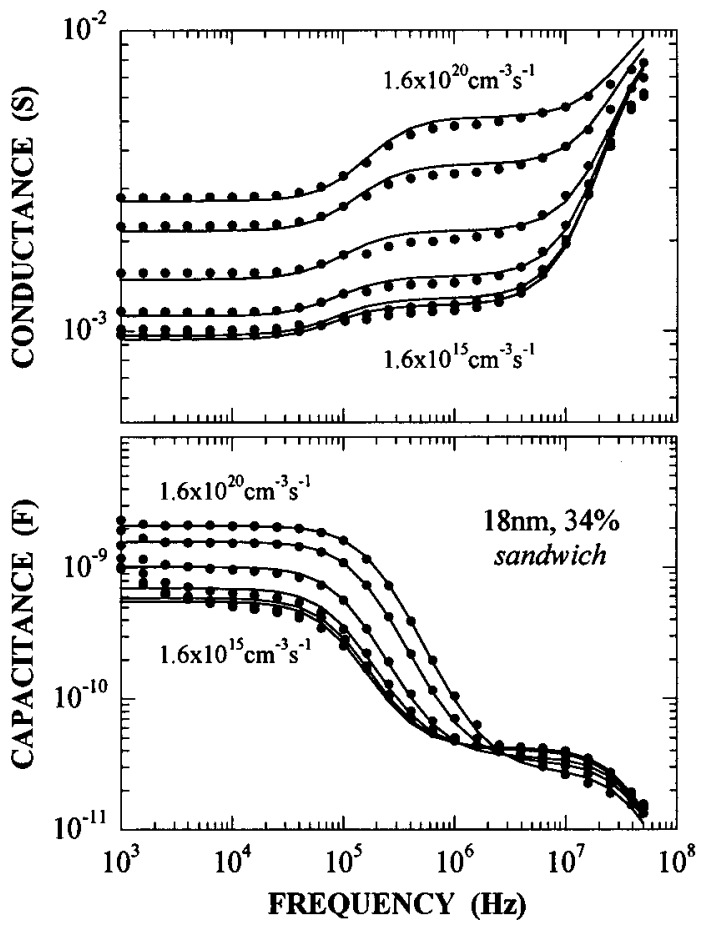

FIG. 4. Frequency spectra of conductance and capacitance measured for the sandwich structure at various generation rates in the range $\bar{g}=1.6$ $\times 10^{15}-1.6 \times 10^{20} \mathrm{~cm}^{-3} \mathrm{~s}^{-1}$. The crystalline grain size $\delta_{(220)}$ and volume fraction $\chi_{c}$ are indicated in the figure. The solid lines are theoretical fits to the data in accordance with the equivalent circuit model.

considered are localized states at which traveling carriers can be trapped as well as the potential barrier over which carriers propagate, both existing in the grain boundary region. It is apparent that they act as an obstacle for intergrain carrier passage, and affect more seriously transport through a percolation cluster formed with small grains having a large surface-to-volume ratio. These considerations give a qualitative interpretation for the observation that the drift mobility increases with the volume fraction and decreases for smaller grains.

\section{B. Perpendicular transport}

\section{Photoadmittance}

Prior to discussing the results of drift mobility experiments in the sandwich electrode arrangement, we will explain the admittance measured under steady-state illumination. Typical frequency spectra of conductance and capacitance observed for various generation rates $\bar{g}$ are shown in Fig. 4. A staircase-like structure is clearly found in the spectra. The conductance remains constant and low in the low frequency region, and then increases with frequency to reach an intermediate step. In the high frequency region, the conductance once again increases with frequency. An enhanced but reversed behavior is seen for the capacitance spectra. A similar feature was found in the previous admittance study. ${ }^{3}$

The peculiar spectral behavior might be ascribed directly to a random mixture of crystalline phase and amorphous phase. A most useful approach to such a problem is the effective-medium theory that calculates the response of an

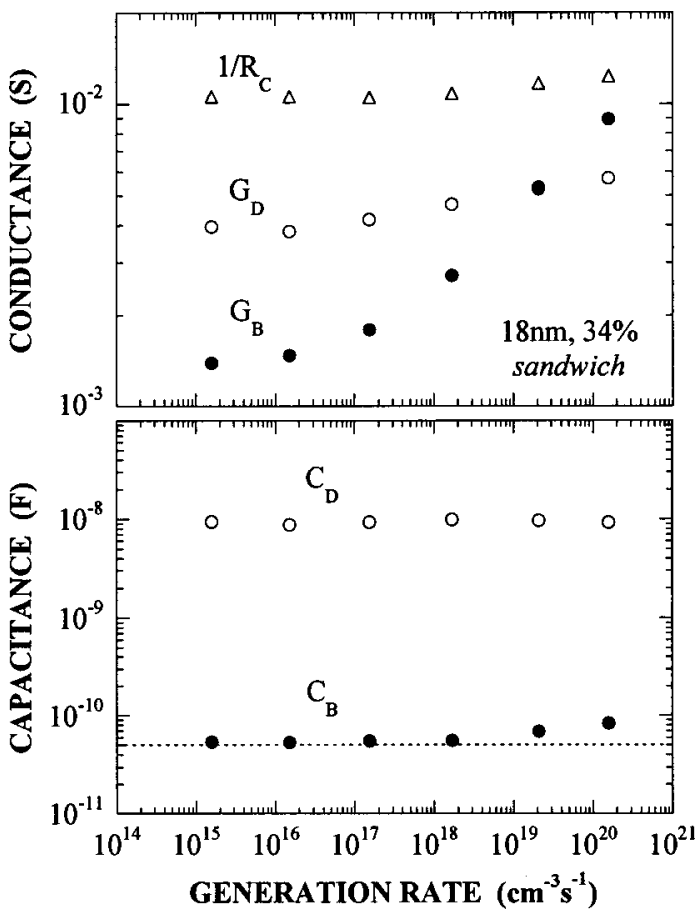

FIG. 5. Generation rate dependence of circuit parameters derived from the theoretical fits shown in Fig. 4. The dotted line indicates the geometrical capacitance for a dielectric constant of 12 .

inhomogeneous medium to an external electric field, by treating the material as a homogeneous substance with an effective dielectric function. ${ }^{24-26}$ The theoretical treatment is reviewed in the Sec. IV A, in association with a formulation of drift mobility for $\mu c-\mathrm{Si}: \mathrm{H}$. In this work, we have compared experimental results with those of effective-medium calculations for the conductance and capacitance spectra. The calculation replicates the step-like spectral structure, however, it is always rather broadened and thereby does not fit the experimental result. This discrepancy suggests that a dominant mechanism of a different kind underlies the observation.

A simpler explanation for the admittance spectra has been introduced by Kocka et al. ${ }^{3}$ They consider the equivalent circuit of a sample composed of a series combination of admittances: $Y_{D}(\Omega)=G_{D}+i \Omega C_{D}$, which represents the depletion layer near the contact, and $Y_{B}(\Omega)=G_{B}+i \Omega C_{B}$, which represents the remaining volume of the film, and of the contact resistance of the electrodes: $R_{C}$. Here, $\Omega$ denotes the angular frequency of the alternating current (ac) voltage. The total admittance of the sample to be observed is then $Y_{\text {tot }}=Y /\left(1+Y R_{C}\right)$, where $Y=Y_{B} Y_{D} /\left(Y_{B}+Y_{D}\right)$. The leastsquare fits to the data are plotted as solid lines. As shown in the figure, the measurements are reasonably well accounted for by this model. The circuit parameters obtained from this procedure are summarized in Fig. 5 as a function of generation rate. Both capacitance components $C_{B, D}$ are almost independent of generation rate. The bulk layer capacitance $C_{B}$ coincides with its geometrical value (shown by the dotted line) calculated with a dielectric constant of 12 , which is typical for Si material irrespective of its structure. In contrast, the depletion layer capacitance $C_{D}$ is extremely large, 


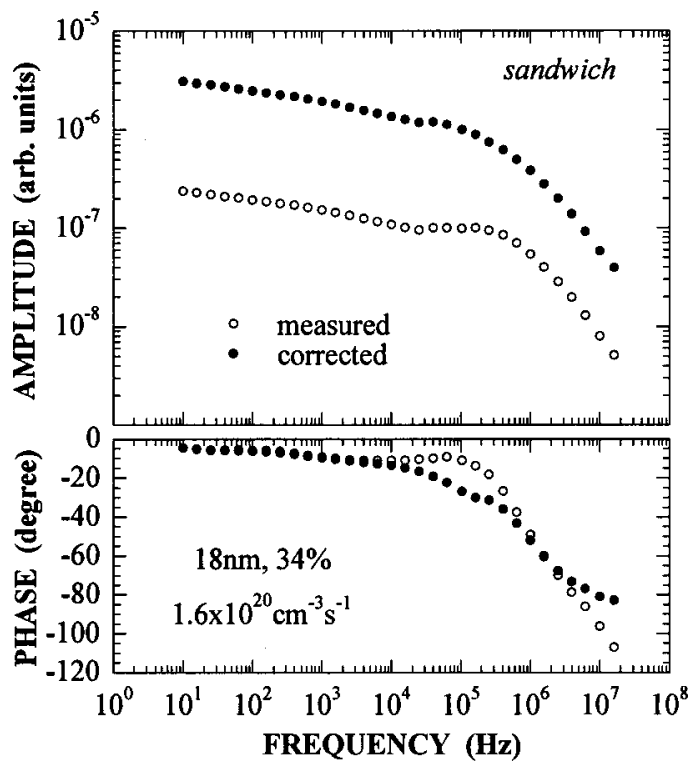

FIG. 6. Comparison between the measured external response $\widetilde{I}(\omega) / V(\bigcirc)$ and the calculated internal response $\widetilde{G}_{B}(\omega)(\bullet)$ in amplitude and phase shift with reference to the modulated photoexcitation. The correction is based on circuit parameters shown in Fig. 5.

and corresponds to a relevant layer thickness as small as 10 $\mathrm{nm}$. The bulk layer conductance $G_{B}$ increases sizably with generation rate, while the depletion layer conductance $G_{D}$ as well as the contact resistance $R_{C}$ show a much weaker dependence. The conductivity of the depletion layer is calculated to be around $5 \times 10^{-7} \mathrm{~S} \mathrm{~cm}^{-1}$, which is orders of magnitude lower than the estimation for the bulk layer which is in the range between $3 \times 10^{-5}$ and $2 \times 10^{-4} \mathrm{~S} \mathrm{~cm}^{-1}$. The physical origin of such a thin layer with a low conductivity is not clear at the present stage of investigation. For the coplanar structure, the numerical simulation indicates that the admittance is little affected by the depletion layer, and behaves approximately as $Y \cong Y_{B}$. This has been confirmed experimentally.

\section{Drift mobility}

The photoadmittance measurements indicate that the photocurrent response of the sample originates mainly from the inner bulk layer. In the MPC experiment, the bulk layer conductance varies with time as $G_{B}(t)=\bar{G}_{B}$ $+\widetilde{G}_{B}(\omega) \exp (i \omega t)$. A small-signal analysis of the equivalent circuit shows that the modulated photocurrent detected on a load resistance $R_{L}$ is expressed as $\widetilde{I}(\omega)$ $=S(0) S(\omega) \widetilde{G}_{B}(\omega) V$, where $V$ is the static voltage applied across the sample, and $S(\omega)=Y(\omega) /\left\{Y_{B}(\omega)\left[1+Y(\omega)\left(R_{C}\right.\right.\right.$ $\left.\left.\left.+R_{L}\right)\right]\right\}$ is the multiplication factor depending on frequency as well as on circuit parameters under steady-state illumination. Figure 6 compares the measured external response $\widetilde{I}(\omega) / V$ and the corrected internal response $\widetilde{G}_{B}(\omega)$, in amplitude and phase shift with reference to the modulated photoexcitation. The amplitude of the external response is smaller than that of the internal response over the entire frequency range, reflecting the fact that only a portion of the applied voltage feeds into the photoconductive bulk layer.
The dielectric relaxation in the depletion layer, the characteristic frequency of which is estimated to be $100 \mathrm{kHz}$, affects the measurement. This shows in the phase-shift bump observed at the relaxation frequency, which disappears in the corrected internal response. At high frequencies, an anomalous phase lag exceeding $90^{\circ}$ is also corrected in this manner.

Deriving the MPC mobility from the modulated photoconductance is a straightforward calculation. The results obtained from $\widetilde{G}_{B}(\omega)$ at $\omega / 2 \pi=10 \mathrm{MHz}$ and $\bar{g}=1-2$ $\times 10^{20} \mathrm{~cm}^{-3} \mathrm{~s}^{-1}$ are summarized in Fig. 3, where the calculations from $\widetilde{I}(\omega) / V$ are also plotted for comparison. The figure illustrates that for all the sandwich samples, the presence of a depletion layer and a resistive contact disturbs the measurement to a significant degree, and the correction is quite substantial for evaluating the MPC mobility that represents the photoconductive region in the sample. To simplify, we shall call it the MPC mobility for sandwich samples in the following. The MPC mobility for sandwich samples containing large grains increases with the crystalline volume fraction, similar to the observation for coplanar structures. This feature is a manifestation of the percolation-controlled transport. A striking difference between sandwich and coplanar structures, in which photocarrier transport is monitored perpendicular and parallel to the substrate, respectively, lies in the magnitude of MPC mobility. The MPC mobility is more than ten times larger for perpendicular transport than parallel transport.

The observation is hardly explained by considering homogeneously distributed spherical crystalline grains. The dominant grains included in our samples are oriented in the [220] direction. It is known that, in such a case, individual grains often have an elongated shape along the growth direction. ${ }^{2}$ Moreover, during film growth, the grains may form a natural cluster with a columnar structure normal to the substrate. ${ }^{1}$ It is then logical to attribute the anisotropy of transport property to the geometry of constituents.

\section{Comparison with previous experiments}

Previously, the drift mobility in undoped $\mu c-\mathrm{Si}: \mathrm{H}$ materials has been investigated by applying various experimental techniques. Before concluding this section it may be appropriate to compare our experimental results with the previous ones. Traveling wave measurements ${ }^{5}$ in the coplanar configuration provided an evaluation of drift mobility in the range $0.1-0.6 \mathrm{~cm}^{2} / \mathrm{V}$ s for samples with $\chi_{c} \cong 75 \%$, which does not differ much from our result. A typical value of 3 $\mathrm{cm}^{2} / \mathrm{V}$ s for sandwich samples was obtained in the study using a method called charge extraction by linearly increasing voltage. ${ }^{8}$ The result is compatible with the present evaluation, in terms of order of magnitude. Time-resolved microwave conductivity experiment ${ }^{9}$ showed that the drift mobility increased from 2 to $9 \mathrm{~cm}^{2} / \mathrm{V} \mathrm{s}$ monotonously with the volume fraction in the range $\chi_{c}=60 \%-90 \%$. The measurement utilizes microwave reflection for monitoring photoconductivity of the film in the absence of external electric field, and largely reflects the motion of carriers in their higher mobility direction. An agreement with our evaluation for perpendicular transport is therefore thought to be reasonable. The larg- 
est disparity is found with the transient grating experiment, ${ }^{6}$ the result of which indicates that the ambipolar drift mobility exceeds $100 \mathrm{~cm}^{2} / \mathrm{V}$ s for parallel transport. It must be borne in mind that this kind of experiment proceeds normally with an extremely intense photoexcitation. It is likely that the resulting high-density nonequilibrium carriers significantly influence electronic properties of the grain (or cluster) boundary region, such as screening of potential barriers or saturation of trap occupancy. These side effects may explain the discrepancy in the measurements.

\section{MODEL CONSIDERATION}

This section presents a theoretical approach to the physical implications from the drift mobility measurements assuming that all the dimensions of the transport regions are greater than the mean free path of carriers. Therefore, the models to be developed are in principle classical systems as far as the effects of disorder and geometry on transport are concerned. Inclusions of both grains and clusters make the theoretical treatment very complex. We simplify the analysis by assuming either of these subsystems to be a limiting factor determining transport properties. In the following we arbitrarily consider the case when grains and their boundaries dominate transport. The discussion for cluster-limited transport is essentially similar if the cluster dimensions are much smaller than the size of the system.

\section{A. Effective-medium model}

The structural inhomogeneity present in disordered materials usually gives significant effects on carrier transport properties. The problem we are dealing with relates to a twophase random system in which a volume fraction of $\chi_{c}$ has a conductivity $\sigma_{c}$ (crystalline phase) and the reminder of $\chi_{a}$ $=1-\chi_{c}$ a conductivity $\sigma_{a}$ (amorphous phase). In that case, the effective-medium theory ${ }^{24,25}$ expresses the static conductivity $\sigma$ of the material as

$$
\sigma=\sigma_{0}+\sqrt{\sigma_{0}^{2}+\sigma_{c} \sigma_{a} / 2}
$$

with $\sigma_{0}=\sum_{i=c, a} \sigma_{i}\left(\chi_{i}-1 / 3\right) /(4 / 3)$. It is easily shown that the expression has a simple limiting form when $\sigma_{a} / \sigma_{c} \rightarrow 0$ : $\sigma=\sigma_{c} \eta\left(\chi_{c}\right)$, where the function $\eta(x)$ is written as $\eta(x)$ $=(x-1 / 3) \theta(x-1 / 3) /(2 / 3)$ with the unit step function $\theta(x)$. This produces a linear increase of $\sigma / \sigma_{c}$ when $\chi_{c} \geqslant 1 / 3$. The critical value, $1 / 3$, is very close to the percolation threshold, $0.31-0.32$, obtained from the numerical computation for a simple cubic network. ${ }^{24,27}$ As mentioned in Sec. III B 1, the effective medium theory can calculate the dielectric function that describes the response of the system to ac electric field. In this article, we do not go into incorporating the ac theory, ${ }^{26}$ being consistent with the interpretation of admittance spectra, and we base our discussion on Eq. (5) and its extension.

The theoretical formulation is easily extended to the modulated part superposed on the steady-state photoconductivity, giving

$$
\widetilde{\sigma}(\omega)=\sum_{i=c, a} s_{i} \widetilde{\sigma}_{i}(\omega),
$$

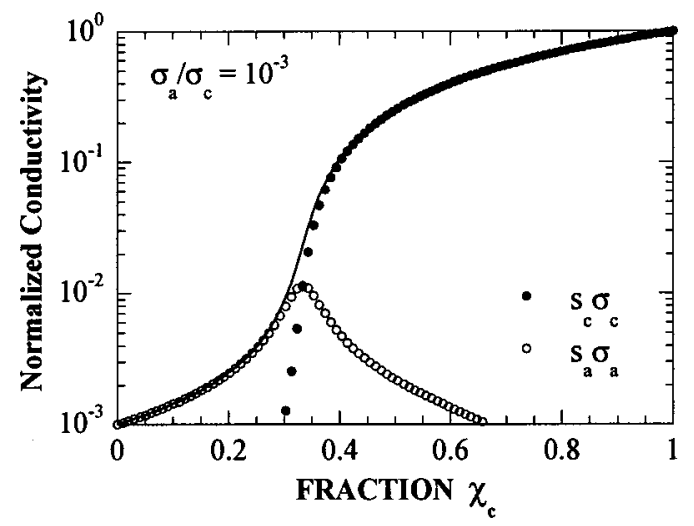

FIG. 7. Effective-medium conductivity $\sigma$ (solid line) and its decomposition into $s_{c} \sigma_{c}$ and $s_{a} \sigma_{a}$ (symbols) as a function of $\chi_{c}=1-\chi_{a}$. In the calculation, the conductivity ratio is chosen as $\sigma_{a} / \sigma_{c}=10^{-3}$.

with the coefficient describing a sensitivity of relevant constituent

$$
s_{i}=\frac{3}{4} \frac{\bar{\sigma}\left(\chi_{i}-1 / 3\right)+\bar{\sigma}_{j} / 3}{\bar{\sigma}-\bar{\sigma}_{0}},
$$

where $j=a, c$ for $i=c, a$. It is noted that an equivalent decomposition, $\bar{\sigma}=\sum_{i=c, a} s_{i} \bar{\sigma}_{i}$, exists for the steady-state conductivity. The volume fraction dependence of the effectivemedium conductivity is illustrated in Fig. 7. Analogous to the general result, one finds that $\widetilde{\sigma}(\omega) \cong \widetilde{\sigma}_{c}(\omega) \eta\left(\chi_{c}\right)$ for a negligibly small conductivity of the amorphous phase. Recall that the MPC experiments indicate a substantial accumulation of photocarriers into the crystalline phase. The approximation mentioned above is therefore practically validated for our $\mu c-\mathrm{Si}: \mathrm{H}$ samples. Consequently, the high-frequency MPC mobility is formulated as

$$
\mu_{\mathrm{MPC}}(\omega)=\mu_{c}(\omega) \eta\left(\chi_{c}\right) / \chi_{c},
$$

where $\mu_{c}(\omega)$ denotes the drift mobility in the crystalline phase. The factor $\chi_{c}^{-1}$ describes the correction for photocarrier density.

The carrier flow in the crystalline phase is considered to be limited by localized states and potential barriers built up at grain boundaries. In order to make the discussion concrete, we shall assume that electrons are the predominant type of carriers in photoconduction. The theoretical analysis on the basis of the Shockley-Read statistics, which postulates that the localized states exchange carriers via the extended band states, yields a concise expression

$$
\mu_{c}(\omega) \cong \frac{\mu_{n}^{f}}{1+\int d \varepsilon N(\varepsilon) \gamma_{n}(\varepsilon, \omega)} .
$$

Here, $\mu_{n}^{f}$ is the mobility of free electrons in the conduction band, and $N(\varepsilon)$ the density of localized states at an energy $\varepsilon$. The function $\gamma_{n}(\varepsilon, \omega)$ describes the modulation of the occupation function due to electron trapping, the details of which are explained in the Appendix. When the electron diffusion length is larger or comparable to the length dimension of grain $\delta$, the function $N(\varepsilon)$ given in Eq. (9) is regarded as the areal density of grain-boundary states multiplied by the surface-to-volume ratio, which generally increases for 
smaller grains. Equation (9) accounts for the contribution from potential barriers, with the effective mobility, $\mu_{n}^{f}$ $=\nu_{\mathrm{th}} P / E_{\mathrm{th}}$, following from the thermionic field emission model. ${ }^{28,29}$ Here, $\nu_{\text {th }}=\sqrt{k T / 2 \pi m}$ is the thermal velocity, and $E_{\mathrm{th}}=k T / q \delta$ the thermal field, expressed with the thermal energy $k T$ and the effective mass of electron $m$. The factor $P$ represents the barrier transmission probability involving two possible channels, classical thermionic emission and quantum mechanical tunneling. In the purely classical case, $P$ $=\exp \left(-\phi_{b} / k T\right)$ for a barrier height of $\phi_{b}$. The theoretical description presented here demonstrates that the boundarylimited drift mobility $\mu_{c}$ correlates with the dimension of grains, and tends to lower for smaller grains.

The parallel drift mobility data show a lower value for smaller grains even at a similar volume fraction, implying that the geometrical constraints are substantial. From a series of drift mobility data obtained for samples with large grains, it has been confirmed that the product $\chi_{c} \mu_{\text {MPC }}$ plotted versus $\chi_{c}$ forms a straight line. The onset of the line is found to be $\chi_{c} \cong 30 \%$ for both parallel and perpendicular mobility data. This behavior is in agreement with that expected from Eq. (8) if $\mu_{c}$ is independent of $\chi_{c}$. The positive correlation between $\delta$ and $\chi_{c}$ observed for our samples, however, implies that $\mu_{c}$ should increase with $\chi_{c}$, contrary to the observation. This makes us reconsider the geometrical effects. In accordance with Seto model, ${ }^{28}$ the potential barrier is produced by the space charge formed near the grain boundary, and the barrier height increases with the grain size when the associated interfacial traps are unsaturated. A structural strain in $\mu c-\mathrm{Si}: \mathrm{H}$, which is thought to be relieved by the inclusion of an amorphous phase, becomes highly concentrated in the grain boundary region with increasing crystalline volume fraction. This may be accompanied by the occurrence of a lot of localized states. These additional mechanisms might operate to compensate the possible variation of $\mu_{c}$ with $\chi_{c}$.

As evident in Eq. (5), the effective-medium theory considers a homogeneous and isotropic substitutive system, and thereby cannot deal with transport anisotropy. In this sense, the argument given above is still oversimplified. Thus, although the present consideration does not provide a rigorous description of carrier transport in $\mu c-\mathrm{Si}: \mathrm{H}$ it still helps understanding the percolation process involved.

\section{B. Random-walk model}

Transport anisotropy itself might be accounted for from a different point of view. The simplest model for $\mu c-\mathrm{Si}: \mathrm{H}$ with a sufficiently high crystalline volume fraction is an array of crystalline grains. A good example for analyzing carrier transport is then a Brownian particle on the threedimensional lattice, where each lattice site is placed at the center of the grains, and the jump of a particle to a nearestneighbor site is limited by grain boundary. Because of the asymmetrical shape of grains, we suppose here, a simple tetragonal lattice with spacings $a=b<c$, where the $c$-axis is parallel to the main axis of the grains. In that case, it follows from elemental statistical considerations that the diffusion front of particles generally forms a spheroid. The relevant diffusion coefficient in the direction labeled by $l=a, b, c$ is simply expressed as $D_{l}=w_{l} l^{2}$, where $w_{l}$ is the probability of jump in the $l$-direction per unit time. This gives an anisotropy represented by the ratio $D_{c} / D_{a, b}=(c / a)^{2}$ for an isotropic jump probability. Assuming that the jump probability is proportional to a contact area such that $w_{c} \propto a b$, one obtains the estimate that $D_{c} / D_{a, b}=c / a$. Since the factor $c / a$ corresponds to the aspect ratio of the grain, the boundary-limited random-walk model reasonably links grain geometry to transport anisotropy.

Although the random-walk model is instructive for understanding transport anisotropy, it does not incorporate the concept of percolation at the present level. To be more general, one has to consider a percolation lattice, where only a fraction of all sites is occupied by grains, the rest being empty. A test particle can move only from an occupied site of the lattice to a nearest neighbor that is also occupied. In this sense, both effective-medium and random-walk models discussed in this article are complementary to each other but clearly insufficient. We believe that a more generalized and sophisticated theory could be derived from extensions of these two basic models.

\section{EVALUATION OF LOCALIZED STATES}

\section{A. Similarity to amorphous silicon}

So far, we have examined the transport mechanism in $\mu c$-Si:H mainly from the drift mobility magnitude measured under a specific condition that $\omega / 2 \pi=10 \mathrm{MHz}$ and $\bar{g}$ $=1-2 \times 10^{20} \mathrm{~cm}^{-3} \mathrm{~s}^{-1}$. In this section, we discuss the frequency spectra observed at various generation rates, as well as the physical insight derived from them. Typical spectra measured for $\mu c-\mathrm{Si}: \mathrm{H}$ are displayed in Fig. 8, and are compared with the results obtained for $a-\mathrm{Si}: \mathrm{H}$. At first sight, one finds that the spectral feature as well as the generation-rate dependence are qualitatively similar for both materials. Each spectrum shows roughly two branches separated by a knee. The frequency at which the knee appears shifts toward the higher frequency side with increasing generation rate. Since the relevant frequency is determined by the average lifetime as described in Sec. II B, the observation is reasonably interpreted in terms of the recombination via localized states populated with nonequilibrium carriers. The high frequency spectra tend to approach a single line, independently of the generation rate. This feature is more clearly identified on the results for $a-\mathrm{Si}: \mathrm{H}$. Conversely, an intermediate part between the low-frequency falloff and the high-frequency convergence varies, depending on the generation rate. The magnitude of MPC mobility in this region increases appreciably with the generation rate, so that the spectra cross one another.

\section{B. Numerical calculation}

\section{Model}

The similarity between $\mu c-\mathrm{Si}: \mathrm{H}$ and $a-\mathrm{Si}: \mathrm{H}$ led us to carry out a detailed theoretical investigation of the MPC spectra on the basis of the distribution of localized states suggested for $a$-Si:H. For this purpose, we have performed a numerical calculation. The calculation incorporates the density of states (DOS) consisting of a distribution of dangling 


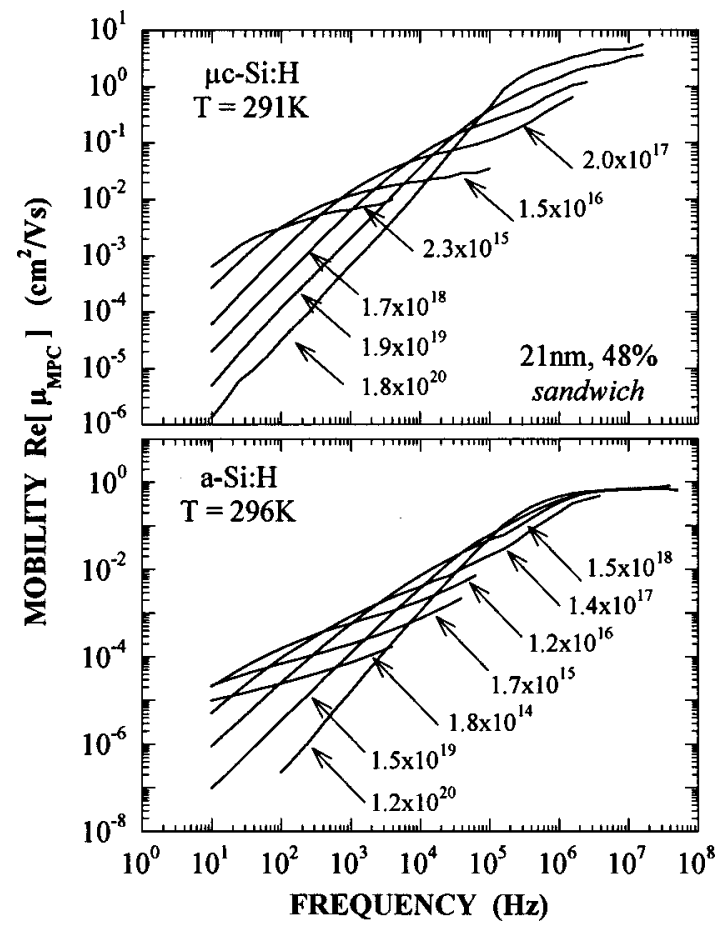

FIG. 8. Frequency spectra of MPC mobility observed for $\mu c-\mathrm{Si}: \mathrm{H}$ (top) and $a-\mathrm{Si}: \mathrm{H}$ (bottom) at various generation rates $\bar{g}$. The mobility data for $\mu c-\mathrm{Si}: \mathrm{H}$ were obtained from the measurements in the sandwich configuration, and corrected in accordance with the equivalent circuit model. The crystalline grain size $\delta_{(220)}$ and volume fraction $\chi_{c}$ of the sample are indicated.

bond (DB) states superposed on band tail (BT) states. The BT-DOS $\left(N^{(\mathrm{BT})}\right)$ decays linearly with a slope of $0.1 \mathrm{eV}$ from the respective band edge and thereafter decreases exponentially toward midgap. ${ }^{14,15}$ The characteristic temperatures describing the exponential parts were chosen as $T_{c}=220 \mathrm{~K}$ for the conduction BT states and $T_{\nu}=500 \mathrm{~K}$ for the valence BT states. A single Gaussian band with a standard deviation 0.1 $\mathrm{eV}$ centered at $0.85 \mathrm{eV}$ from the valence band edge $\varepsilon_{\nu}$ was assumed for the DB-DOS $\left(N^{(\mathrm{DB})}\right)$. The correlation energy of $\mathrm{DB}$ states was taken as $U=0.3 \mathrm{eV}$. In thermal equilibrium, singly occupied (neutral) DB occupation prevails within the energy interval between the Fermi level $\varepsilon_{F}$ and $\varepsilon_{F}-U$. The DB states above $\varepsilon_{F}$ are mainly unoccupied (positively charged) while the states below $\varepsilon_{F}-U$ are doubly occupied (negatively charged). Charge neutrality in equilibrium is approximately maintained by an equal integrated density of positively and negatively charged DB states, positioning the Fermi level at $\varepsilon_{F}=\varepsilon_{\nu}+1 \mathrm{eV}$. The total DB density was adjusted so as to give the neutral DB density of $3 \times 10^{15} \mathrm{~cm}^{-3}$ at $T=300 \mathrm{~K}$, which is a typical value for $a-\mathrm{Si}: \mathrm{H}$. At this temperature, the charged-to-neutral defect density ratio was calculated to be 0.15 .

The rate equations for the nonequilibrium occupation functions of DB states, $f^{+}$(positively charged), $f^{0}$ (neutral), and $f^{-}$(negatively charged), are expressed with the densities of free electrons $n^{f}$ and holes $p^{f}$ as

$$
\begin{aligned}
\frac{\partial}{\partial t} f^{+}(\varepsilon, t)= & {\left[p^{f}(t) C_{p}^{0}+e_{n}^{0}(\varepsilon)\right] f^{0}(\varepsilon, t) } \\
& -\left[n^{f}(t) C_{n}^{+}+e_{p}^{+}(\varepsilon)\right] f^{+}(\varepsilon, t),
\end{aligned}
$$

$$
\begin{aligned}
& \frac{\partial}{\partial t} f^{-}(\varepsilon, t)= {\left[n^{f}(t) C_{n}^{0}+e_{p}^{0}(\varepsilon)\right] f^{0}(\varepsilon, t) } \\
&-\left[p^{f}(t) C_{p}^{-}+e_{n}^{-}(\varepsilon)\right] f^{-}(\varepsilon, t), \\
& f^{+}(\varepsilon, t)+f^{0}(\varepsilon, t)+f^{-}(\varepsilon, t)=1 .
\end{aligned}
$$

The electron occupancy of BT states obeys

$$
\begin{aligned}
\frac{\partial}{\partial t} f(\varepsilon, t)= & {\left[n^{f}(t) C_{n}+e_{p}(\varepsilon)\right][1-f(\varepsilon, t)] } \\
& -\left[p^{f}(t) C_{p}+e_{n}(\varepsilon)\right] f(\varepsilon, t) .
\end{aligned}
$$

In the expressions given above, $e_{n, p}$ are the thermal emission rates for an electron and a hole from BT states, and $C_{n, p}$ are the associated capture coefficients. The carrier emission rates $e_{n}^{0,-}$ and $e_{p}^{+, 0}$ and the capture coefficients $C_{n}^{+, 0}$ and $C_{p}^{0,-}$ correspond to the DB states, where the superscripts denote charge states as usual. Combining the rate equations with the continuity equations for free carriers

$$
\begin{aligned}
\frac{d}{d t} n^{f}(t)= & g(t)-\int d \varepsilon N^{(\mathrm{BT})}(\varepsilon)\left\{n^{f}(t) C_{n}[1-f(\varepsilon, t)]\right. \\
& \left.-e_{n}(\varepsilon) f(\varepsilon, t)\right\}-\int d \varepsilon N^{(\mathrm{DB})}(\varepsilon) \\
& \times\left\{n^{f}(t) C_{n}^{+} f^{+}(\varepsilon, t)+\left[n^{f}(t) C_{n}^{0}-e_{n}^{0}(\varepsilon)\right]\right. \\
& \left.\times f^{0}(\varepsilon, t)-e_{n}^{-}(\varepsilon) f^{-}(\varepsilon, t)\right\} \\
\frac{d}{d t} p^{f}(t)= & g(t)-\int d \varepsilon N^{(\mathrm{BT})}(\varepsilon)\left\{p^{f}(t) C_{p} f(\varepsilon, t)\right. \\
& \left.-e_{p}(\varepsilon)[1-f(\varepsilon, t)]\right\}-\int d \varepsilon N^{(\mathrm{DB})}(\varepsilon) \\
& \times\left\{p^{f}(t) C_{p}^{-} f^{-}(\varepsilon, t)+\left[p^{f}(t) C_{p}^{0}-e_{p}^{0}(\varepsilon)\right]\right. \\
& \left.\times f^{0}(\varepsilon, t)-e_{p}^{+}(\varepsilon) f^{+}(\varepsilon, t)\right\},
\end{aligned}
$$

the solutions for free carrier densities and occupation functions were obtained for a modulated photoexcitation $g(t)$ $=\bar{g}+\widetilde{g}(\omega) \exp (i \omega t) .{ }^{11,30}$ The MPC mobility discussed below was calculated assuming that photocurrent is dominated by electrons. The charged DB state may have a higher capture efficiency than a neutral one due to Coulomb attraction. To examine this effect on the MPC mobility, the capture coefficient ratio, $r_{\text {cap }}=C_{n}^{+} / C_{n}^{0}=C_{p}^{-} / C_{p}^{0}$, was varied from 1 to 100 , while $C_{n, p}^{0}$ was kept fixed at $10^{-8} \mathrm{~cm}^{3} \mathrm{~s}^{-1}$. The capture coefficients $C_{n, p}$ for BT states were assumed to be identical to $C_{n, p}^{0}$.

\section{Result}

The results of the calculations are summarized in Fig. 9, where the MPC mobility $\operatorname{Re}\left[\mu_{\mathrm{MPC}}\right]$ is displayed topographically as a function of frequency $\omega / 2 \pi$ and generation rate $\bar{g}$. The lines correspond to a sequence of mobility values spaced in equal logarithmic steps. The graph is shaded in such a way that regions with higher values are lighter. Three distinctive regions can be observed in the contour map of MPC mobility. At sufficiently high frequencies, the electron transport is mainly controlled by trapping at the conduction BT states. 


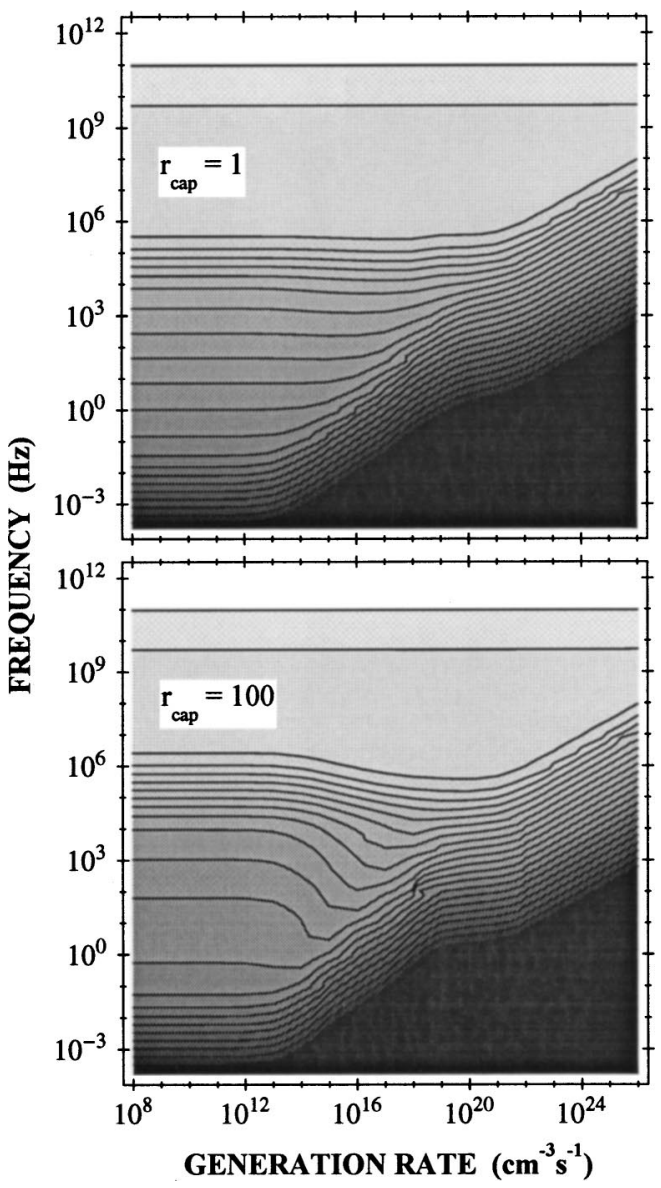

FIG. 9. Topographic illustration of MPC mobility calculated for $r_{\text {cap }}=1$ (top) and 100 (bottom) as a function of frequency and generation rate. The lines contour mobility values spaced in equal logarithmic steps $(\log [10] / 2)$. The graph is shaded in such a way that regions with higher values are lighter.

Since they are distributed near the conduction band edge and have high thermal-emission rates, a quasithermal equilibrium with the conduction band tends to be established promptly. This results in a drift mobility being weakly dependent on frequency, and a plateau-like topography in the corresponding area. The recombination-limited falloff forms a valley in the area with a low frequency and a high generation rate. A hill sloping gradually toward the lower frequency side is found in the area with a low generation rate, which is mainly due to trapping at deep DB states. A significant difference between the results obtained for $r_{\text {cap }}=1$ (top) and for $r_{\text {cap }}$ $=100$ (bottom) is found in a medium range of generation rates. For the higher capture coefficient ratio, the BT plateau extends to a lower frequency region, particularly for generation rates ranging from $\bar{g}=10^{18}$ to $10^{22} \mathrm{~cm}^{-3} \mathrm{~s}^{-1}$. Simultaneously, the DB hill grows for $\bar{g}=10^{14}-10^{18} \mathrm{~cm}^{-3} \mathrm{~s}^{-1}$. The observation in the contour map is clearly confirmed in Fig. 10 , which displays a collection of frequency spectra for $\bar{g}$ $=10^{13}-10^{19} \mathrm{~cm}^{-3} \mathrm{~s}^{-1}$. The calculation for $r_{\text {cap }}=1$ shows no crossing of the spectra, as opposed to that for $r_{\text {cap }}=100$. The latter reproduces well the spectral behavior observed in the experiment. From the numerical results, this behavior is interpreted as being a characteristic signature of a higher capture efficiency for charged DB states than neutral ones. ${ }^{11,12}$

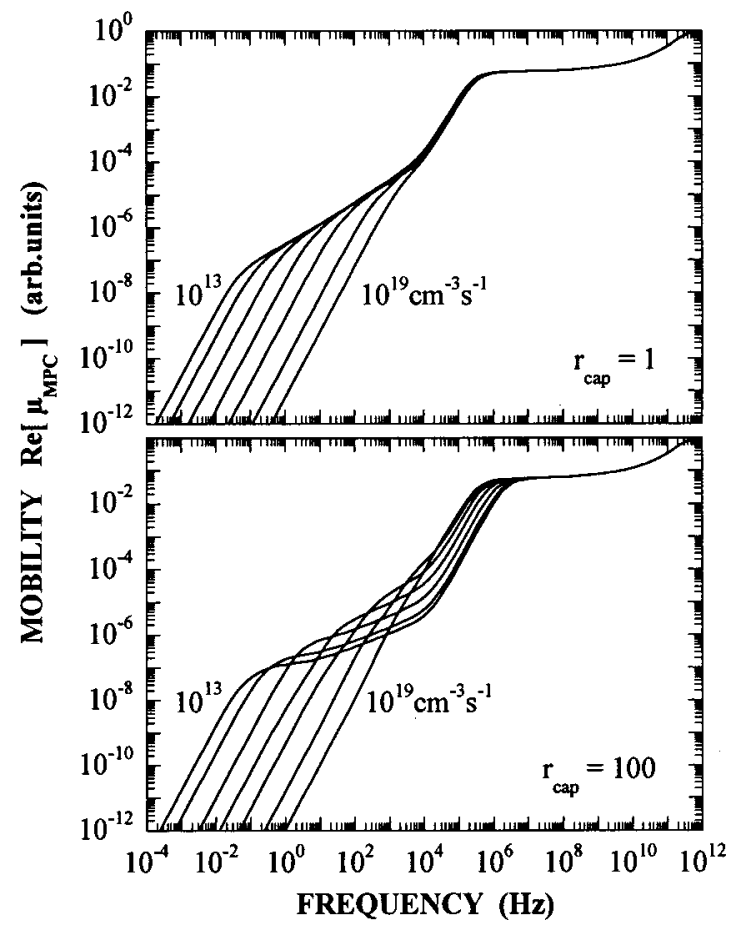

FIG. 10. Frequency spectra of MPC mobility calculated for $r_{\text {cap }}=1$ (top) and 100 (bottom). The seven curves plotted in each figure correspond to generation rates varying from $10^{13}$ to $10^{19} \mathrm{~cm}^{-3} \mathrm{~s}^{-1}$ in 1 order of magnitude steps.

The present experiment is therefore an indication that both $a-\mathrm{Si}: \mathrm{H}$ and $\mu c-\mathrm{Si}: \mathrm{H}$ materials contain such defects commonly.

It is well known that the MPC experiment provides a useful means not only to measure the photocarrier drift mobility but also to evaluate the DOS distributions as well as the capture coefficients. The results of such measurements are discussed in the next subsection, and confirm a similarity between defects in $a-\mathrm{Si}: \mathrm{H}$ and $\mu c-\mathrm{Si}: \mathrm{H}$, in a more quantitative way.

\section{Density of states and capture coefficient}

Based on Eqs. (10)-(12), the behavior of modulated photoconductivity related to the coexistence of BT and DB states has been theoretically studied in previous works. ${ }^{11,12,30}$ The analysis derives a high-frequency approximation such that

$$
\begin{aligned}
& \operatorname{Im}\left[\widetilde{g}(\omega) / \widetilde{n}^{f}(\omega)\right] \\
& \cong \omega+C_{n} \int_{\varepsilon_{t n}}^{\varepsilon_{c}} d \varepsilon N^{(\mathrm{BT})}(\varepsilon) \operatorname{sech}\left[\left(\varepsilon-\varepsilon_{\omega}\right) / k T\right] / 2 \\
& \quad+C_{n}^{+} \int_{\varepsilon_{t n}^{0}}^{\varepsilon_{c}} d \varepsilon N^{(\mathrm{DB})}(\varepsilon) \operatorname{sech}\left[\left(\varepsilon-\varepsilon_{\omega}^{+/ 0}\right) / k T\right] / 2 \\
& \quad+C_{n}^{0} \int_{\varepsilon_{t n}^{-}}^{\varepsilon_{t n}^{0}} d \varepsilon N^{(\mathrm{DB})}(\varepsilon) \bar{f}^{0}(\varepsilon) \operatorname{sech}\left[\left(\varepsilon-\varepsilon_{\omega}^{0 /-}\right) / k T\right] / 2 .
\end{aligned}
$$

It is noted that the ratio $\widetilde{g}(\omega) / \widetilde{n}^{f}(\omega)$ on the left-hand side of the equation is equal to $i \omega \mu_{n}^{f} / \mu_{\mathrm{MPC}}(\omega)$ for $\widetilde{\sigma}(\omega)$ 


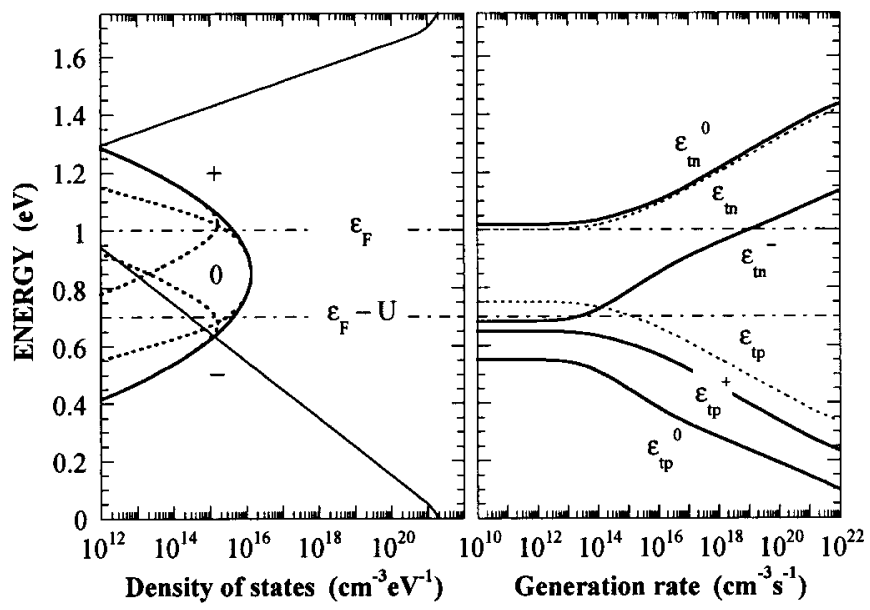

FIG. 11. Model density-of-states (left side) and generation rate dependence of quasi-Fermi levels for trapped carriers (right) calculated for $r_{\text {cap }}=100$. Three components of charge states $(+, 0$, and -$)$ in equilibrium at $T$ $=300 \mathrm{~K}$ are plotted as dotted lines along with the DB distribution.

$=q \mu_{n}^{f} \widetilde{n}^{f}(\omega)$. The equality may not be valid for the MPC mobility measured for $\mu c-\mathrm{Si}: \mathrm{H}$, but the proportionality between $\widetilde{g}(\omega) / \widetilde{n}^{f}(\omega)$ and $i \omega / \mu_{\mathrm{MPC}}(\omega)$ is reasonably assumed, within the framework of the effective-medium model discussed in Sec. IV A. The frequency-dependent emission energies, $\varepsilon_{\omega}$, $\varepsilon_{\omega}^{+/ 0}$, and $\varepsilon_{\omega_{0}}^{0 /-}$, and the quasi-Fermi levels for trapped electrons, $\varepsilon_{t n}, \varepsilon_{t n}^{0}$, and $\varepsilon_{t n}^{-}$, are defined as

$$
\begin{aligned}
& \varepsilon_{\omega}=\varepsilon_{c}-k T \ln \left(N_{c} C_{n} / \omega\right), \\
& \varepsilon_{\omega}^{+/ 0}=\varepsilon_{c}-k T \ln \left(N_{c} C_{n}^{+} / 2 \omega\right), \\
& \varepsilon_{\omega}^{0 /-}=\varepsilon_{c}-k T \ln \left(2 N_{c} C_{n}^{0} / \omega\right)-U,
\end{aligned}
$$

and

$$
\begin{aligned}
& \varepsilon_{t n}=\varepsilon_{c}-k T \ln \left[N_{c} C_{n} /\left(\bar{n}^{f} C_{n}+\bar{p}^{f} C_{p}\right)\right], \\
& \varepsilon_{t n}^{0}=\varepsilon_{c}-k T \ln \left[N_{c} C_{n}^{+} / 2\left(\bar{n}^{f} C_{n}^{+}+\bar{p}^{f} C_{p}^{0}\right)\right], \\
& \varepsilon_{t n}^{-}=\varepsilon_{c}-k T \ln \left[2 N_{c} C_{n}^{0} /\left(\bar{n}^{f} C_{n}^{0}+\bar{p}^{f} C_{p}^{-}\right)\right]-U,
\end{aligned}
$$

where $N_{c}$ is the effective density of conduction band states above $\varepsilon_{c}$. The generation rate dependence of these quasiFermi levels is illustrated in Fig. 11, together with the DOS distribution assumed for calculation.

The first term $\omega$ on the right hand side of Eq. (13) is usually neglected when compared with the last three terms that represent the effects of electron trapping at the conduction BT states and the DB states. Each term consists of the DOS distribution broadened by convoluting a hyperbolic secant function with a width of $k T$ and a peak shifting with $\omega$. The DB contribution is made of a mixture coming from different energy intervals, $\varepsilon_{t n}^{0}<\varepsilon<\varepsilon_{c}$ (third term) and $\varepsilon_{t n}^{-}<\varepsilon$ $<\varepsilon_{t n}^{0}$ (fourth term). The possible mixing is generally a source of uncertainty in the analysis of experimental results by means of Eq. (13). In this regard, the inference that $C_{n}^{+}$ $\gg C_{n}^{0}$ permits a useful and realistic approximation. The inequality suggests a larger contribution from the third term than from the fourth term, and hence simplifies Eq. (13) into

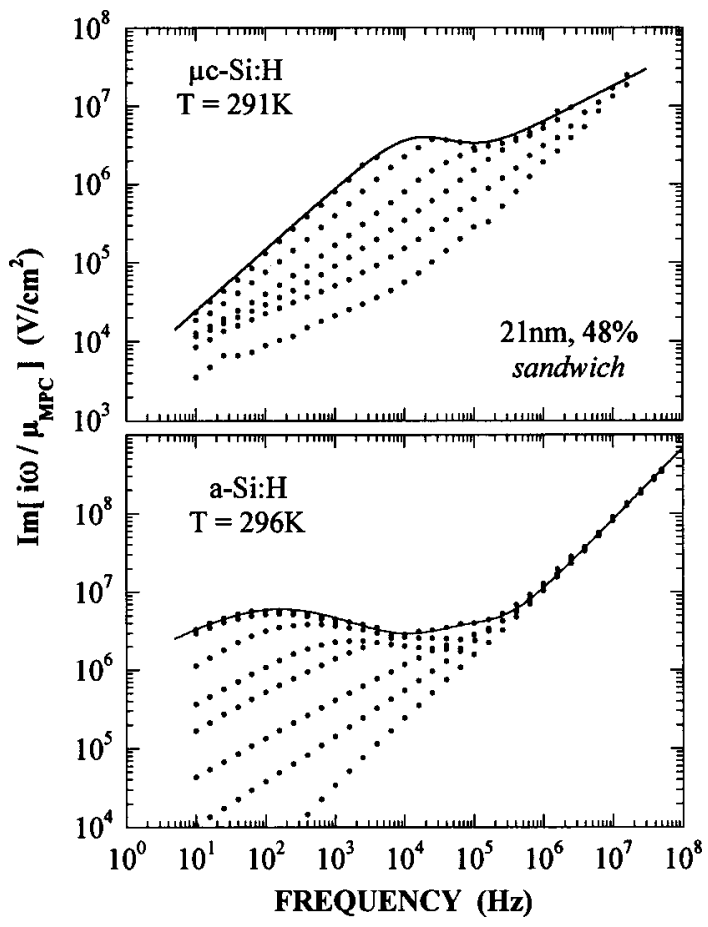

FIG. 12. Frequency dependence of $\operatorname{Im}\left[i \omega / \mu_{\mathrm{MPC}}\right]$ for $\mu c-\mathrm{Si}: \mathrm{H}$ (top) and $a$-Si:H (bottom). Each curve corresponds to the result for the different generation rate shown in Fig. 8. The solid line represents a merger of the high frequency parts.

$$
\begin{aligned}
& \operatorname{Im}\left[\widetilde{g}(\omega) / \widetilde{n}^{f}(\omega)\right] \\
& \cong C_{n} \int_{\varepsilon_{t n}}^{\varepsilon_{c}} d \varepsilon N^{(\mathrm{BT})}(\varepsilon) \operatorname{sech}\left[\left(\varepsilon-\varepsilon_{\omega}\right) / k T\right] / 2 \\
& \quad+C_{n}^{+} \int_{\varepsilon_{t n}^{0}}^{\varepsilon_{c}} d \varepsilon N^{(\mathrm{DB})}(\varepsilon) \operatorname{sech}\left[\left(\varepsilon-\varepsilon_{\omega}^{+/ 0}\right) / k T\right] / 2 .
\end{aligned}
$$

The validity of Eq. (14) has been confirmed by the model calculation with $r_{\text {cap }}=100$. Namely, the approximation for $\operatorname{Im}\left[\widetilde{g}(\omega) / \widetilde{n}^{f}(\omega)\right]$ is justifiable, consistently with the replication of the spectral features shown by $\operatorname{Re}\left[\mu_{\mathrm{MPC}}\right]$.

Figure 12 displays the frequency dependence of $\operatorname{Im}\left[i \omega / \mu_{\mathrm{MPC}}\right]$ calculated from the experimental data for $\mu c-\mathrm{Si}: \mathrm{H}$ and $a-\mathrm{Si}: \mathrm{H}$ samples. Each curve corresponds to the result for the different generation rates shown in Fig. 8. The curve exhibits a falloff in a low frequency region where the modulated photoconductivity is controlled by recombination, similar to the observation for $\operatorname{Re}\left[\mu_{\mathrm{MPC}}\right]$. The curves for different generation rates join at high frequencies where Eq. (14) is reasonably applied. The connection of the high frequency parts thus outlines a linear combination of the thermally broadened DOS distributions. The distribution so determined (plotted as a solid line) exhibits a power-law curve at high frequencies and a bump at low frequencies. In terms of Eq. (14), they are ascribed to contributions from the conduction BT states and the DB states, respectively.

The quasi-Fermi level $\varepsilon_{t n}^{0}$ is located at $\varepsilon_{F}+k T \ln (2)$ for sufficiently low generation rates. As for a Gaussian DBDOS shown in Fig. 11, this means that the peak of DBrelated bump occurs when $\omega \cong n_{\mathrm{eq}}^{f} C_{n}^{+}$, where $n_{\mathrm{eq}}^{f}$ is the free electron density in thermal equilibrium. For our $a-\mathrm{Si}: \mathrm{H}$ 
sample, the equilibrium electron density was calculated to be $n_{\mathrm{eq}}^{f} \approx 10^{8} \mathrm{~cm}^{-3}$ from the dark conductivity, yielding an estimated value of $C_{n}^{+} \approx 10^{-5}-10^{-6} \mathrm{~cm}^{3} \mathrm{~s}^{-1}$ for the peak frequency $\omega / 2 \pi \approx 100 \mathrm{~Hz}$. On the other hand, for $\mu c-\mathrm{Si}: \mathrm{H}$, assuming the equilibrium-Fermi level to be lying at center of the band gap ${ }^{17}$ corresponds to $n_{\mathrm{eq}}^{f} \approx 10^{10} \mathrm{~cm}^{-3}$. Then, the peak observed at $\omega / 2 \pi \approx 10 \mathrm{kHz}$ leads us to conclude that the $C_{n}^{+}$value for $\mu c-\mathrm{Si}: \mathrm{H}$ is comparable to that for $a-\mathrm{Si}: \mathrm{H}$. This confirms that DB defects in both materials have a very similar character.

\section{SUMMARY AND CONCLUSIONS}

We have investigated photocarrier transport and the localized states in undoped $\mu c-\mathrm{Si}: \mathrm{H}$ by means of the MPC technique. The experiments show that the photocarrier drift mobility varies systematically with the crystalline grain size, the volume fraction, as well as the transport direction. The drift mobility measured in the coplanar electrode configuration increases with the volume fraction while a lower mobility is observed for smaller grains. The measurement in the sandwich arrangement reveals that the drift mobility in the direction perpendicular to the substrate is 1 order of magnitude higher than parallel to the substrate. The observations indicate that transport takes place via a grain-to-grain carrier delivery, and reflects the connectivity and the geometry of grains included in the material. These effects are discussed with two basic models. The effective-medium model brings into light the percolation process involved in the transport, and satisfactorily accounts for the drift mobility increase with the volume fraction observed in the experiment. The lower drift mobility for smaller grains proves that the grain boundary bottlenecks the percolation transport. The boundary-limited random-walk model reasonably links the grain geometry to the transport anisotropy. Apart from the variation of drift mobility magnitude, the frequency spectra and generation rate dependence are also extensively discussed in comparison with those of $a-\mathrm{Si}: \mathrm{H}$. The comparative study suggests that DB states contained in these materials have a very similar character. Based on the numerical simulation as well as on a direct evaluation from the measurements, we interpret the experimental results as indicating that common to both materials, the charged DB state possesses a higher capture efficiency than neutral ones.

\section{ACKNOWLEDGMENT}

The authors greatfully acknowledge Dr. T. Matsui (National Institute of Advanced Industrial Science and Technology, Japan) for supplying $\mu c$-Si:H samples which were prepared in his work at the Graduate School of Engineering Science, Osaka University.

\section{APPENDIX: ANALYSIS OF SHOCKLEY-READ EQUATIONS}

For the sake of simplicity, we consider a single species of monovalent states with an energy distribution $N(\varepsilon)$, through which electronic transitions occur at rates $n^{f}(t) C_{n}$, $p^{f}(t) C_{p}, e_{n}(\varepsilon)$, and $e_{p}(\varepsilon)$. According to the ShockleyRead formalism, the rate equation for electron occupation function $f(\varepsilon, t)$ is given by Eq. (11). The continuity equations for free carriers $n^{f}(t)$ and $p^{f}(t)$ are expressed as Eqs. (12a) and (12b), respectively, replacing $N^{(\mathrm{BT})}(\varepsilon)$ by $N(\varepsilon)$ and equating $N^{(\mathrm{DB})}(\varepsilon)$ to zero. It is easy to calculate the steady-state occupation function, which is written as

$$
\bar{f}(\varepsilon)=\left[\bar{n}^{f} C_{n}+e_{p}(\varepsilon)\right] / \rho(\varepsilon),
$$

with the sum of transition rates $\rho(\varepsilon)=\bar{n}^{f} C_{n}+\bar{p}^{f} C_{p}+e_{n}(\varepsilon)$ $+e_{p}(\varepsilon)$. The modulated part of the occupation function involves two components relative to electrons and holes

$$
\widetilde{f}(\varepsilon, \omega)=\widetilde{n}^{f}(\omega) \gamma_{n}(\varepsilon, \omega)-\widetilde{p}^{f}(\omega) \gamma_{p}(\varepsilon, \omega),
$$

where the coefficients $\gamma_{n, p}(\varepsilon, \omega)$ are expressed as

$$
\begin{aligned}
& \gamma_{n}(\varepsilon, \omega)=C_{n}[1-\bar{f}(\varepsilon)] /[i \omega+\rho(\varepsilon)], \\
& \gamma_{p}(\varepsilon, \omega)=C_{p} \bar{f}(\varepsilon) /[i \omega+\rho(\varepsilon)] .
\end{aligned}
$$

In terms of Eq. (A2), it is natural to express the trapped carrier densities as

$$
\begin{aligned}
& \widetilde{n}^{t}(\omega)=\widetilde{n}^{f}(\omega) \Gamma_{n}(\omega), \\
& \widetilde{p}^{t}(\omega)=\widetilde{p}^{f}(\omega) \Gamma_{p}(\omega),
\end{aligned}
$$

with the energy integrals

$$
\Gamma_{n, p}(\omega)=\int d \varepsilon N(\varepsilon) \gamma_{n, p}(\varepsilon, \omega) .
$$

The total electron and hole densities must satisfy the charge neutrality, which is exactly shown by the following equality: $\widetilde{n}(\omega)=\widetilde{n}^{f}(\omega)+\widetilde{n}^{t}(\omega)=\widetilde{p}^{f}(\omega)+\widetilde{p}^{t}(\omega)$. The drift mobility $\mu(\omega) \quad$ defined as $\widetilde{\sigma}(\omega)=q\left[\mu_{n}^{f} \widetilde{n}^{f}(\omega)+\mu_{p}^{f} \widetilde{p}^{f}(\omega)\right]$ $=q \mu(\omega) \widetilde{n}(\omega)$ is explicitly given by

$$
\mu(\omega)=\frac{\mu_{n}^{f}}{1+\Gamma_{n}(\omega)}+\frac{\mu_{p}^{f}}{1+\Gamma_{p}(\omega)} .
$$

Note that the electron-related term in Eq. (A6) coincides with the right hand side of Eq. (9). On the other hand, the average lifetime defined as $\widetilde{n}(\omega) / \widetilde{g}(\omega)=1 /[i \omega+1 / \tau(\omega)]$ is found to be

$$
\frac{1}{\tau(\omega)}=\frac{1}{\left[1+\Gamma_{n}(\omega)\right] \tau_{n}(\omega)}+\frac{1}{\left[1+\Gamma_{p}(\omega)\right] \tau_{p}(\omega)},
$$

where

$$
\begin{aligned}
& 1 / \tau_{n}(\omega)=\int d \varepsilon N(\varepsilon) \gamma_{n}(\varepsilon, \omega)\left[\bar{p}^{f} C_{p}+e_{p}(\varepsilon)\right], \\
& 1 / \tau_{p}(\omega)=\int d \varepsilon N(\varepsilon) \gamma_{p}(\varepsilon, \omega)\left[\bar{n}^{f} C_{n}+e_{n}(\varepsilon)\right] .
\end{aligned}
$$

The analysis of Eqs. (10)-(12) is somewhat tedious though the calculation itself does not present any mathematical difficulties. Basically, it consists of the solution of a system of six equations for the same number of unknown quantities. The calculation leads to similar formulations of drift mobility and average lifetime, in which $\Gamma_{n, p}(\omega)$ and $1 / \tau_{n, p}(\omega)$ are expressed as linear combinations of contributions from BT and DB states such that $\Gamma_{n, p}(\omega)=\sum_{i=\mathrm{BT}, \mathrm{DB}} \Gamma_{n, p}^{(i)}(\omega)$ and $1 / \tau_{n, p}(\omega)=\Sigma_{i=\mathrm{BT}, \mathrm{DB}} 1 / \tau_{n, p}^{(i)}(\omega)$. 
${ }^{1}$ M. Luysberg, P. Hapke, R. Carius, and F. Finger, Philos. Mag. A 75, 31 (1997).

${ }^{2}$ N. Wyrsch et al., Mater. Res. Soc. Symp. Proc. 609, A15.1 (2000).

${ }^{3}$ J. Kocka, A. Fejfar, V. Vorlicek, H. Stuchlikova, and J. Stuchlik, Mater.

Res. Soc. Symp. Proc. 557, 483 (1999).

${ }^{4}$ T. Unold, R. Bruggemann, J. P. Kleider, and C. Longeaud, J. Non-Cryst. Solids 266-269, 325 (2000).

${ }^{5}$ S. Huang, L. Wang, G. Ganguly, J. Xu, X. Huang, A. Matsuda, and K. Chen, J. Non-Cryst. Solids 266-269, 347 (2000).

${ }^{6}$ J. Kudrna, F. Trojanek, P. Maly, and I. Pelant, Appl. Phys. Lett. 79, 626 (2001).

${ }^{7}$ V. Svrcek et al., J. Appl. Phys. 89, 1800 (2001).

${ }^{8}$ G. Juska, K. Arlauskas, N. Nekrasas, J. Stuchlik, X. Niquille, and N. Wyrsch, J. Non-Cryst. Solids 299-302, 375 (2002).

${ }^{9}$ F. Liu, M. Zhu, Y. Feng, Y. Han, J. Liu, S. Kasouit, and R. Vanderhaghen, J. Non-Cryst. Solids 299-302, 385 (2002).

${ }^{10}$ N. Wyrsch, C. Droz, L. Feitknecht, P. Torres, E. Vallat-Sauvain, J. Bailat, and A. Shah, J. Non-Cryst. Solids 299-302, 390 (2002).

${ }^{11}$ K. Hattori, Y. Adachi, M. Anzai, H. Okamoto, and Y. Hamakawa, J. Appl. Phys. 76, 2841 (1994).

${ }^{12} \mathrm{~K}$. Hattori, H. Okamoto, and Y. Hamakawa, J. Non-Cryst. Solids 198200, 288 (1996).

${ }^{13}$ K. Hattori, T. Hirao, M. Iida, and H. Okamoto, J. Non-Cryst. Solids 266269, 352 (2000).

${ }^{14}$ K. Hattori, M. Iida, T. Hirao, and H. Okamoto, J. Appl. Phys. 87, 2901 (2000).
${ }^{15}$ K. Hattroi, T. Hirao, Y. Musa, and H. Okamoto, Phys. Rev. B 64, 125208 (2001).

${ }^{16}$ K. Hattori, Y. Musa, and H. Okamoto, J. Non-Cryst. Solids 299-302, 546 (2002).

${ }^{17}$ T. Matsui, R. Muhida, T. Kawamura, T. Toyama, H. Okamoto, T. Yamazaki, S. Honda, H. Takakura, and Y. Hamakawa, Appl. Phys. Lett. 81, 4751 (2002)

${ }^{18}$ E. Bardet, J. E. Bouree, M. Cuniot, J. Dixmier, P. Elkaim, J. L. Duigou, A. R. Middya, and J. Perrin, J. Non-Cryst. Solids 198-200, 867 (1996).

${ }^{19}$ T. Tiedje, in Semiconductors and Semimetals, edited by J. I. Pankove (Academic, New York, 1984), Vol. 21C, pp. 207-238.

${ }^{20}$ D. Ritter, E. Zeldov, and K. Weiser, Phys. Rev. B 38, 8296 (1988).

${ }^{21}$ K. Hattori, H. Okamoto, and Y. Hamakawa, Phys. Rev. B 45, 1126 (1992).

${ }^{22}$ K. Hattori, Y. Koji, S. Fukuda, W. Ma, H. Okamoto, and H. Hamakawa, J. Appl. Phys. 73, 3846 (1993).

${ }^{23}$ D. Stauffer and A. Aharony, Introduction to Percolation Theory (Routledge, London, 1994).

${ }^{24}$ S. Kirkpatrick, Rev. Mod. Phys. 45, 574 (1973).

${ }^{25}$ B. E. Springett, J. Appl. Phys. 44, 2925 (1973).

${ }^{26}$ B. E. Springett, Phys. Rev. Lett. 31, 1463 (1973).

${ }^{27}$ H. Overhof, M. Otte, M. Schmidtke, U. Backhausen, and R. Carius, J. Non-Cryst. Solids 227-230, 992 (1998).

${ }^{28}$ J. Y. W. Seto, J. Appl. Phys. 46, 5247 (1975).

${ }^{29}$ T. Weis, R. Lipperheide, U. Wille, and S. Brehme, J. Appl. Phys. 92, 1411 (2002).

${ }^{30}$ C. Longeaud and J. P. Kleider, Phys. Rev. B 48, 8715 (1993). 